\title{
Formal Mathematics for Mathematicians
}

\section{Foreward to the Special Issue}

\author{
Andrzej Trybulec • Artur Kornilowicz • Adam Naumowicz • \\ Krystyna Kuperberg
}

Received: 9 October 2012 / Accepted: 10 October 2012 / Published online: 1 November 2012

(C) The Author(s) 2012. This article is published with open access at Springerlink.com

\begin{abstract}
The collection of works for this special issue was inspired by the presentations given at the 2011 AMS Special Session on Formal Mathematics for Mathematicians: Developing Large Repositories of Advanced Mathematics. The issue features a collection of articles by practitioners of formalizing proofs who share a deep interest in making computerized mathematics widely available.
\end{abstract}

Keywords Formalization • Repositories of mathematics • Mathematical proof reconstruction

\section{Introduction}

It has been shown in recent years that proof checkers and theorem provers can be successfully used by mathematicians for automated proving and formal verification of non-trivial results. One key to this success was the development of formalisms close to the so-called "mathematical vernacular" in a way acceptable by mathematicians and software capable of processing such input. Numerous attempts have been made to make the mathematical community more interested in all these developments, to name just the four excellent papers that appeared in Notices of

\footnotetext{
A. Trybulec $\cdot$ A. Kornilowicz · A. Naumowicz $(\varangle)$

Department of Mathematics and Informatics, University of Bialystok, Bialystok, Poland e-mail: adamn@math.uwb.edu.pl
}
A. Trybulec e-mail: trybulec@math.uwb.edu.pl

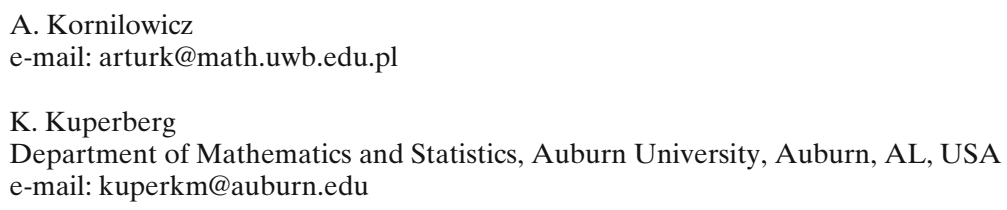


the AMS in 2008 [4-6, 10], describing non-trivial examples developed by working mathematicians, as well as how working mathematicians could get started using the most popular systems available. There are also several conferences and workshops organized by the community of formal proof developers with the aim to make the idea of computerized formalization of mathematical proofs more widely recognized. Most notably, let us mention here the yearly events that gather both practitioners and theorists of formal proofs by presenting the on-going research and current trends in this area under the umbrella of Conferences on Intelligent Computer Mathematics $[1,3,7]$ and Interactive Theorem Proving [2, 8, 9]. Still, most mathematicians are probably not aware that the third revolution in mathematics (as Wiedijk called the introduction of computer formalized mathematics [10]) has already begun and now there is a chance to extend mathematics with new results and possibilities.

Hence, there came to existence the idea of organizing the AMS Special Session on Formal Mathematics for Mathematicians: Developing Large Repositories of Advanced Mathematics at the AMS Joint Mathematics Meetings held in New Orleans, USA in January 2011. The session gathered developers of several proof checking systems and some others working on proof theory. The inspiring presentations and discussions gave rise to this special issue of the Journal of Automated Reasoning.

\section{Contents of the Issue}

Amongst the crucial problems in collecting large formalized mathematics data are efficient methods of accumulating knowledge, so that new developments can be based on top of previously proved results. This issue is addressed in the paper by J. Harrison, showing how a comprehensive HOL Light library was developed based on the concept of $n$-dimensional Euclidean spaces. In a similar spirit, the article by Y. Futa, H. Okazaki and Y. Shidama describes the development of elliptic curve systems with applications in cryptoghraphic systems using Mizar.

For the formalized mathematics to successfuly advance the mathematical practice, it is necessary to further develop formalisms with emphasis on computer verified rigorous proofs that can be expressive enough to suit the needs of mathematicians and enable practical computer encoding. Important contributions into this area are the works by J. Belinfante describing the GOEDEL program and the paper on the formalization of theories by J. Mycielski.

The development of existing libraries shows that creating large repositories of advanced mathematics requires research on effective semantic-based knowledge management to facilitate the process of maintaining the library and offering users better interfaces and presentation formats. In their paper, J. Urban, P. Rudnicki and G. Sutcliffe describe their work on improving the experience of authoring formalizations by employing artificial intelligence and automated theorem proving tools. That work is also closely related to the aspects of presentation and interoperability of formalized knowledge, which is a central of point of J. Urban's joint paper with M. Iancu, M. Kohlhase and F. Rabe.

The existing libraries of formalized mathematics are sufficiently large to allow drawing conclusions about the structure of proofs, definitions and whole theories. The papers by J. Alama and K. Pask analyze and present methods to optimize the existing body of interrelated mathematics accumulated in formal repositories. 
Interacting with a huge library is, however, becoming a more and more difficult task for the users of proof assistants. Some examples of efficient techniques to reduce the effort of formalizing mathematics are the main topics of the article by M. Caminati and G. Rosolini, as well as the paper by A. Korniłowicz.

The collection of articles reveals many similarities between the systems used for the presented developments, but also significant differences. We believe that it is an important challenge to identify the best of today's achievements and to integrate them into a single best practice environment. We feel confident that within this issue the reader can find relevant papers that will further promote the potential of formal mathematics to members of the mathematical community.

We would like to use this opportunity to express our gratitude to all research groups and researchers who participated in the New Orleans meeting, and especially those who submitted their contributions and made this special issue possible. We are also very grateful to all the reviewers for their professional and responsible work.

Open Access This article is distributed under the terms of the Creative Commons Attribution License which permits any use, distribution, and reproduction in any medium, provided the original author(s) and the source are credited.

\section{References}

1. Autexier, S. et al. (eds.): Intelligent Computer Mathematics. LNAI 6167. Springer (2010)

2. Beringer, L., Felty, A. (eds.): Interactive Theorem Proving-Third International Conference. LNCS 7406. Springer (2012)

3. Davenport, J., et al. (eds.): Intelligent Computer Mathematics. LNAI 6824. Springer (2011)

4. Gonthier, G.: Formal proof-the four color theorem. Not. Am. Math. Soc. 55(11), 1382-1393 (2008)

5. Hales, T.C.: Formal proof. Not. Am. Math. Soc. 55(11), 1370-1380 (2008)

6. Harrison, J.: Formal proof-theory and practice. Not. Am. Math. Soc. 55(11), 1395-1406 (2008)

7. Jeuring, J., et al. (eds.): Intelligent Computer Mathematics. LNAI 7362. Springer (2012)

8. Kaufmann, M., Paulson, L.C. (eds.): Interactive Theorem Proving, First International Conference. LNCS 6172. Springer (2010)

9. van Eekelen, M.C.J.D., et al. (eds.): Interactive Theorem Proving-Second International Conference. LNCS 6898. Springer (2011)

10. Wiedijk, F.: Formal Proof-Getting Started. Not. Am. Math. Soc. 55(11), 1408-1414 (2008) 